\title{
Edutainment Robotics as Learning Tool
}

\author{
Eleonora Bilotta, Lorella Gabriele, Rocco Servidio, and Assunta Tavernise \\ Linguistics Department, University of Calabria, \\ via P. Bucci, Cube 17/B, 87036 Arcavacata di Rende, Cosenza, Italy \\ \{bilotta, lgabriele, servidio, tavernise\} @unical.it
}

Extended summary. Many constructivist technologies, such as Lego® MindStorms ${ }^{\mathrm{TM}}$ robotic kit, allow students to improve problem solving strategies in educational settings, encouraging teamwork and creativeness. This latter Edutainment robotic kit has been built in accordance with learning principles derived from Piaget and Vygotskij theories of cognitive development, as revised by Papert, which portray learning as the acquisition or 'construction' of knowledge through observation of the effects of one's actions on the world.

In this work we focused on cognitive strategies adopted by University students attending a Robotics laboratory program, working in an Edutainment setting with the Lego MindStorms kit. We analyzed the students' reports on the teamwork during laboratory activities, and we gathered information on the subjects' working modalities during the activities of building and programming basic robots. First of all, we detected three different typologies of work subdivision: each member adopted a role in the building work and all programmed the robot; members did not adopt a fixed rule in building and programming the robot; each member adopted a role in building and programming the robot and a leader supervised the work. We then found that the use of robots stimulated students to explore their own knowledge in a critical way and to share it within the group, and that the activity of realizing an artefact took place through precise phases. These phases were:

1) Planning and building of the artefact, related to problem identification and objective definition, collection and production of ideas, problem conceptualization. During the building and manipulation phases, a fundamental role was exercised by the perceptive and behavioral functions and by the affordance that the elements of the kit suggested to the subjects. As they progressed with the work, students advanced in the learning process and became able to explore, arrange and recombine, in different ways, the material structures and the creative ideas in order to realize the final artefact.

2) Behavioral programming, in which students detected problems, hypothesized and applied solution strategies. In this way, they were able to enrich their work through new details, looking at different thinking modalities; in particular, in planning strategies students divided the problem into many parts and elaborated each part from the particular to arrive at a general solution. This phase was strictly related to the check phase.

3) Check, in which subjects evaluated the realization of the artefact and decided on the need to go back to the building phase, to the programming phase, to both, or rather to search for new ideas.

Thanks to the results of this analysis we can affirm that the Robotics laboratory stimulated students with regard to problem finding (the subject identifies and formulates the initial idea or the problem to solve), problem solving (the subject elaborates and explores some possible solutions in order to reach the objective) and checking procedures (the subject evaluates the artefact's properties from functional, planning and behavioral points of view). The repetition of each of these phases allowed the subjects to modify and improve the structure and this reflected their mental model of the artefact in relation to the assignment. Edutainment Robotics creates a cognitive bridge between educational aims and concrete experience, encouraging in the students, at different levels, the acquisition of new skills in an engaging setting. 